Verschluss des persistierenden Foramen ovale- der letzte Schluss?

Markun, Stefan

DOI: https://doi.org/10.1024/1661-8157/a001322

Other titles: Closure of persistent foramen ovale - the last closure?

Posted at the Zurich Open Repository and Archive, University of Zurich ZORA URL: https://doi.org/10.5167/uzh-90681

Journal Article

Accepted Version

Originally published at:

Markun, Stefan (2013). Verschluss des persistierenden Foramen ovale- der letzte Schluss? Praxis, 102(13):815-816.

DOI: https://doi.org/10.1024/1661-8157/a001322 


\section{Verschluss des persistierenden Foramen ovale - der letzte Schluss?}

\section{Frage:}

Ist der Verschluss eines persistierenden Foramen ovale (PFO) einer nur medikamentösen Sekundärprävention bei vermuteten paradoxen Thromboembolien überlegen?

\section{Hintergrund:}

Arterielle Embolien können bei PFO durch Gerinnsel verursacht werden, die im venösen System entstanden sind (sog. paradoxe Embolien). Dieser Zusammenhang ist im Einzelfall bei idiopathischen Thromboembolien häufig schwer zu beweisen, allerdings wurde die Reduktion von zerebrovaskulären Insulten durch einen Verschluss des PFO in solchen Situationen bereits in Studien bewiesen. Der gesamthafte Nutzen des PFO Verschlusses wird aufgrund von möglichen Komplikationen des Eingriffes in Metaanalysen allerdings infrage gestellt. Diese Studie untersucht, ob ein PFO Verschluss der rein medikamentösen Behandlung überlegen ist bezüglich des Verhinderns von allen Arten von thromboembolischen Rezidiven.

\section{Einschlusskriterien:}

- Patienten $<60$ Jahre alt

- PFO mit nachgewiesenem rechts-links Shunt in der transösophagealen Echokardiographie.

- Ischämischer zerebrovaskulärer Insult (CVI) oder transitorische ischämische Attacke (TIA), jeweils gesichert durch ein neuroradiologisches Korrelat oder klinisch und radiologisch nachgewiesene extrakranielle Thromboembolien

\section{Ausschlusskriterien:}

- Nachweis anderer Ursachen für eine Thromboembolie (spezifische kardiogene, vaskuläre, inflammatorische und hämatologische Ursachen mussten gemäss einer Checkliste ausgeschlossen werden).

- Status nach bereits durchgeführtem PFO Verschluss

- Spezifische Komorbiditäten

\section{Studiendesign und Methode:}

Randomisierte, multizentrische Überlegenheitsstudie

\section{Studienort:}

Zentren in Europa, Kanada, Brasilien und Australien

Interventionen:

- Gruppe 1: Perkutaner kathetertechnischer Verschluss des PFO (mittels Amplatzer PFO Occluder)

- Gruppe 2: Medikamentöse Behandlung (ohne genaues Protokoll, Antiaggregation oder Antikoagulation je nach dem Dafürhalten des behandelnden Arztes)

\section{Outcome:}

Primärer outcome

- Zusammengezählte Häufigkeit von Todesfällen, CVI, TIA und extrakraniellen Thromboembolien. 
- Zusätzlich: Kardiovaskuläre Todesfälle, neue Arrhythmien, Myokardinfarkte, Hospitalisierungen und Probleme im Zusammenhang mit der PFO Behandlung

\section{Resultat:}

- 414 Patienten wurden randomisiert, bei 204 wurde einen PFO Verschluss geplant, bei 210 eine medikamentöse Behandlung. Die Patienten waren durchschnittlich 44 Jahre alt, und wurden im Mittel während 4.1 Jahren in der Studie verfolgt. Die Daten der Patienten wurden in der ursprünglich zugeteilten Gruppe ausgewertet unabhängig von der tatsächlichen Einhaltung der zugeteilten Intervention („intention to treat" Analyse).

- In der Verschlussgruppe konnte bei 188 Patienten erfolgreich ein Verschlussimplantat ins PFO eingelegt werden. Nach 6 Monaten wurde bei 145 dieser Patienten eine transösophageale Echokardiographie durchgeführt, bei $96 \%$ der Patienten fand sich ein anhaltender Verschluss des PFO.

- In der Gruppe, welche der medikamentösen Behandlung zugeteilt war erhielten 200 der 210 randomisierten Patienten tatsächlich eine solche Behandlung. Allerdings entschieden sich 28 dieser Patienten im Verlauf der Studie das PFO doch noch verschliessen zu lassen, sechs von diesen Patienten entschieden aufgrund einer TIA oder eines CVI.

- Der primäre Endpunkt ereignete sich in der Gruppe mit PFO Verschluss in 7 (3.4\%) Patienten, in der Gruppe unter medikamentöser Therapie in 11 (5.2\%) Patienten, dieser Unterschied war statistisch allerdings nicht signifikant $(p=0.34)$.

- Bezüglich Analyse auf sekundäre Endpunkte fanden sich ebenfalls keine signifikanten Unterschiede.

\section{Kommentar:}

- Die Studie vergleicht bei Patienten mit vermuteten paradoxen Embolien die medikamentöse Behandlung mit dem interventionellen Verschluss des PFO. Bezüglich der definierten Endpunkte konnte die Studie keinen signifikanten Nutzen durch einen PFO Verschluss zeigen.

- Die Analyse erfolgte primär nach dem „intention to treat" Prinzip. 14\% der Patienten in der Gruppe der medikamentösen Behandlung liessen sich im Verlauf der Studie allerdings das PFO verschliessen. Diese Patienten wurden somit in der medikamentösen Gruppe ausgewertet, obwohl sie eigentlich einen PFO Verschluss erhalten hatten. Um diesen Störfaktor auszuschliessen wurde eine weitere Analyse durchgeführt, in der nur Patienten berücksichtig wurden, die Ihre ursprünglich zugeteilten Behandlungen wirklich durchgeführt hatten ( "per protocol" Analyse). Auch in dieser Analyse zeigte sich jedoch kein signifikanter Effekt zugunsten des PFO Verschlusses.

- Bei genauer Betrachtung der Zahlen (fünf CVI unter medikamentöser Behandlung versus ein CVI nach PFO Verschluss) kommt die Vermutung auf, dass ein PFO Verschluss möglicherweise trotzdem wirksam sein könnte. Möglicherweise handelt es sich jedoch um einen schwachen Effekt, der sich in dieser Studie aufgrund einer zu geringen Patientenzahl oder einer zu kurzen Studiendauer nicht zeigen liess.

\section{Literatur:}

Meier B, Kalesan B, Mattle HP, et al. Percutaneous closure of patent foramen ovale in cryptogenic embolism. N Engl. J Med. Mar 2013;368:1083-1091

\section{Verfasser:}

Stefan Markun 